\title{
Minor ailment consultations: a mismatch of perceptions between patients and GPs
}

Caroline J. Morris and Judith A. Cantrill School of Pharmacy and Pharmaceutical Sciences, University of Manchester, Manchester, UK and Marjorie C. Weiss Division of Primary Health Care, University of Bristol, Bristol, UK

\begin{abstract}
It is anecdotally reported that general practitioners (GPs) consider much of their time is wasted by patients consulting with minor ailments. The management of minor ailments and the need to reduce pressure on GPs has been a recent focus of government health policy. However, knowledge of the lay and professional interpretations of the term 'minor ailment' is sparse. This study determined GPs' and patients' views of consultations perceived by patients to be for minor ailments. A face-to-face, structured interview was conducted with all consenting patients immediately prior to their consultation with two GPs, over a total of 20 surgery sessions, in England. The GP view of consultations perceived by patients to be for minor ailments was obtained. A total of 240 patients $(96.4 \%$ ) participated in the study. Almost $40 \%$ of interviewees (93/240) indicated that they were consulting about a minor ailment. In many cases the GPs gave a contrasting view of these consultations, with approximately half judged not to be minor ailments. Whilst there was agreement that the remaining consultations were for minor conditions, sometimes a mismatch existed in the perception of whether the consultation was necessary. In almost $30 \%$ of cases $(n=27)$ the GPs viewed that the patient could have managed their condition alone. Policy-makers and GPs maintain that minor ailments account for a substantial proportion of GP workload. However, in this study over $50 \%$ of patients had underestimated their 'minor' condition. Furthermore, two subgroups of minor ailment consultation were identified; those that could and those that could not be managed by the patient alone. Minor ailment consultations would therefore appear to be far more complex than trivial, inappropriate or unnecessary consultations that waste the time of GPs.
\end{abstract}

Key words: general practice; GP consultation; GPs' views; minor ailments; patients' views

\section{Introduction}

It is widely recognized that the incidence of illness and symptoms in the community is high. However, only a small proportion is ultimately presented to the general practitioner (GP) (Bentzen et al., 1989; Freer, 1980; Morrell, 1976; Scambler et al., 1981). Health problems have been conceptualized in the literature as an 'iceberg of illness', those reaching formal medical care being the tip, the rest remain-

Address for correspondence: Caroline Morris, The Drug Usage and Pharmacy Practice Group, School of Pharmacy and Pharmaceutical Sciences, University of Manchester, Oxford Road, Manchester M13 9PL, UK.

Email: Caroline.J.Morris@man.ac.uk ing below the surface (Hannay, 1979). Despite this, it is widely reported, albeit anecdotally, that GPs consider a substantial proportion of their time is wasted by patients consulting with minor ailments. Clearly, the way in which patients manage ailments that they, themselves, perceive as minor has potentially significant implications for GP workload. If patients choose to consult their GP for ailments the GP considers trivial, this may block appointments upon which there is already extreme pressure.

Both the management of minor ailments and the need to alleviate pressure on GPs have been a focus of government health policy in recent years. The rapid expansion of National Health Service (NHS) Direct (Department of Health, 1999a) and 
the introduction of NHS primary care walk-in centres being direct results of these policies (Department of Health, 1999b). Patients are increasingly being encouraged to take greater personal control and responsibility for their own health. In addition, the increasing number of medicines being deregulated from prescription only to pharmacy status has broadened the range of ailments potentially self-treatable by patients. Despite this health policy focus, knowledge of the lay and professional interpretations of the term 'minor ailment' is sparse.

Previous research has tended to focus on inappropriate, trivial or unnecessary consultations (Cartwright, 1967; Cartwright and Anderson, 1981), rather than specifically addressing minor ailments per se. However, specific conditions, perceived as minor ailments from a GP perspective, were identified as forming a subgroup of these consultations (Cartwright, 1967). Furthermore, with the recent developments in health policy, it is not known how relevant these studies are today. It is notable, however, that in some of the more recent research centred on GP workload and the role of the nurse in primary care, minor ailment consultations have, from a research perspective, been considered as synonymous with requests for same day appointments. (Marsh and Dawes, 1995; Shum et al., 2000). However, it is not known from a patient or professional perspective whether this is actually true. The objective of this small study was therefore to determine GPs' and patients' views of consultations perceived by patients to be for minor ailments.

\section{Method}

A short, structured, quantitative interview was conducted by one of the authors (CJM) with all patients consulting one GP from each of two diverse practices. One was a suburban singlehanded practice (practice A) and the other, an urban three-partnered practice (practice B), located in the West Midlands, England. The reception staff recruited patients as they presented for their appointment. Those agreeing to participate were directed to the researcher. Interviews took place in the practice immediately prior to each patient consultation over a period of one week in February
1998 in practice A and May/June 1998 in practice B (a total of 20 surgery sessions). Patients were initially asked if they had experienced any illness in the previous two weeks that they, in their own opinion, considered to be a minor ailment. Those responding positively were asked for brief details about the management of their condition and whether their current consultation was related to this ailment.

In order to explore GPs' views, the participating GPs were asked two closed questions about those consultations perceived by patients to be for minor ailments. First, whether they viewed the condition to be a minor ailment and secondly, whether the patient could have managed the illness episode alone. In order to preserve confidentiality and anonymity, patients were identified by appointment date and time alone. To facilitate data collection and minimize the GPs' time commitment, both GPs elicited the information by reviewing their computerized patient records, in a single session, on completion of the week's data collection from patients.

\section{Results}

\section{Response rate}

During the data collection periods, 249 GP consultations took place (practice A, $n=124$; practice $\mathrm{B}, n=125)$. Eight patients declined to participate in the interview, whilst one patient presenting as an emergency was taken immediately through to the GP and thus excluded. A total of 240 patients (practice A, $n=123$; practice $\mathrm{B}, n=117$ ) were therefore recruited to the study giving an overall response rate of $96.4 \%$.

\section{Patient interview}

Approximately 60\% (145/240) of patients had experienced an ailment that they considered to be minor in the previous two weeks. Of these, just under two-thirds (93/145) volunteered that they were now consulting their GP about this same illness episode. Therefore, overall, almost $40 \%$ (93/249) of all GP consultations that had taken place during the study period were for what the patient considered to be a minor ailment. 


\section{GPs' views}

To allow these data to be placed in context the participating GPs were each asked to give their own definition of 'minor ailment' prior to data collection. Their responses are shown in Figure 1.

Figure 1 Minor ailment definitions

An illness or symptoms that are self-limiting, where the patient could reasonably self-medicate for, if appropriate, and could reasonably be expected to identify themselves. (GP practice $A$ )

An illness that is commonly encountered with which patients are familiar and have previous experience of, or exposure to. One that is dealt with by reassurance and self-medication rather than prescription only medicines. (GP practice $B$ )

It is notable that even though many patients considered that they were consulting about a minor ailment, the GP view was often different. Figure 2 illustrates these data, with consultations viewed as minor ailments subdivided into those which the GPs considered the patient could, or could not, have managed alone.

In almost one-third of cases (27/91) the GPs considered that the consultation was a minor ailment that could have been managed alone. As the GP view was known only for those consultations that patients had considered to be minor ailment consultations this would suggest that the minimum level of unnecessary consultations was $10.8 \%$ (27/249). As some of the unknown visits (i.e., those consultations perceived by patients to be for something other than a minor ailment) would potentially have been perceived by the GPs as unnecessary minor ailment consultations, then the actual level is likely to be higher.

When giving their views on these 91 consultations, for approximately half of the consultations, GPs spontaneously volunteered an explanation for their view that the consultation could or could not have been managed alone. From these data three categories of unnecessary minor ailment consultation were identified. These are shown in Figure 3 together with some illustrative examples.

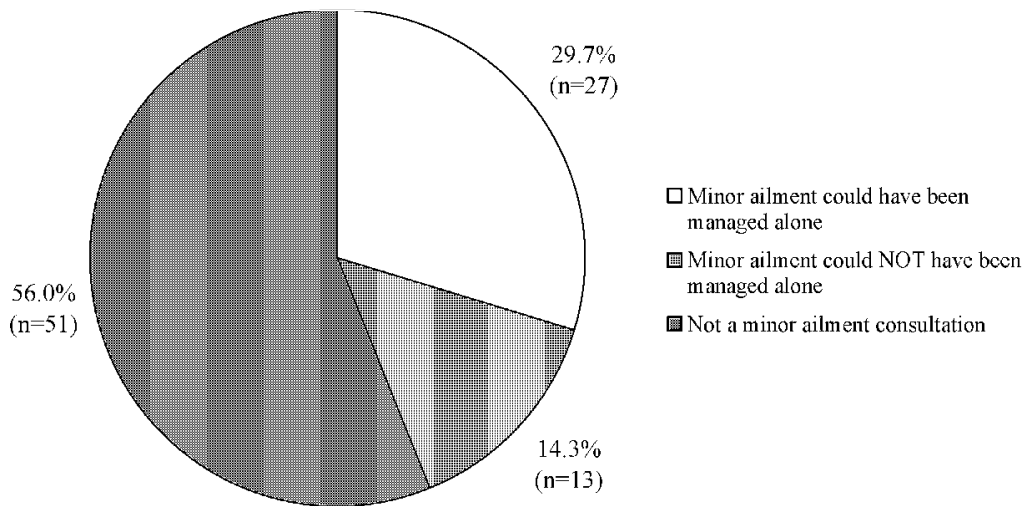

Figure 2 GPs' views of patient perceived minor ailment consultations $(n=91)$. One patient had separate appointments scheduled with the nurse and doctor, yet only consulted the nurse. Data were unavailable for one consultation 
Figure 3 Consultations viewed by the GPs as unnecessary minor ailment consultations

1) Consultations where patients inappropriately demanded antibiotics, e.g. patients with a viral upper respiratory tract infection.

2) Consultations by patients with prior experience of the same minor ailment, e.g. a patient with considerable experience of backache and its management.

3) Consultations for conditions that could be managed with medicines available without a prescription (over-thecounter medicines), e.g. a minor muscle strain where a short course of oral ibuprofen was recommended and consultations for athletes foot, cough and hay fever.

Figure 4 Consultations viewed by the GPs as necessary minor ailment consultations

1) Consultations where treatment could only be provided through the GP surgery, e.g. a patient whose ears required syringing.

2) Consultations where an underlying psychological problem was considered to be of importance, e.g. a patient with diarrhoea and sickness considered to require bereavement counselling and a patient experiencing menorrhagia who needed her cancer fears allaying.

3) Consultations where patients could not reasonably identify the cause of their problem, e.g. a patient with scabies presenting with a skin rash of unknown origin.

Figure 5 Consultations viewed by the GPs not to be minor ailments

1) Patients needing prescription-only medicines, e.g. consultations for coughs, colds or sore throats where sinusitis or a chest infection, needing antibiotic treatment, was diagnosed. Asthmatics with coughs, colds or throat symptoms requiring steroid treatment. Consultations for severe back pain and migraine.

2) Consultations where a full examination was required for accurate symptom assessment, e.g. a consultation for a painful knee which was diagnosed as rheumatoid arthritis. A consultation for a worsening cough where the patient had sel-medicated with terbutaline syrup prescribed for a previous illness episode. On full examination the symptoms were found to be due to an exacerbation of heart failure.

3) Consultations where referral to another professional, only accessible through the GP, was required, e.g. a consultation for recurrent headaches where the patient was referred to a hospital department for full investigation of the symptoms and a patient requiring physiotherapy treatment.
It is notable that in $14.3 \%$ of cases there was complete agreement between the patient and their GP (Figure 2). They both perceived the consultation to be for a minor ailment that could not be managed alone. Data obtained from the GPs suggested three categories of necessary minor ailment consultation. These are shown in Figure 4.

The GPs viewed over half of the patient perceived minor ailment consultations not to be minor ailments (Figure 2). Unsurprisingly, the GPs considered that none of these consultations could have been managed by the patient alone. These consultations fell into the three distinct categories shown in Figure 5.

\section{Discussion}

There are a number of limitations that need to be borne in mind when interpreting the results of this study. First, being a small study, no attempt was made to ensure a representative sample of GP patients. The guiding methodological principle was to ensure that variation existed within the sample by recruiting patients from two diverse practices. The results are therefore likely to reflect features of the individual GPs and their practice base, including how the GPs handle patients consulting with minor ailments, the demographic profile of the practice population and ease of access to GP services. Furthermore, the participating GPs could be considered atypical in that they had agreed to lend their support to this study. The findings cannot therefore be generalized to GPs or their patients as a whole. Secondly, a number of potential sources of bias exist. Although the prevalence of patient perceived minor ailment consultations was high, in reality, it may have been higher. It is possible that some patients may not have been prepared to admit to a researcher to consulting about an ailment that they had just identified as minor. However, sampling GP patients may have over-represented people who are inherently high users of GP services, compared to sampling the general public. There were two further limitations, both imposed by practical constraints. In order to minimize the GPs' time commitment, their views were known only for those consultations that patients perceived to be for a minor ailment. Therefore, GPs' perceptions of only a subset of their consultations were identified, not their view on all consultations taking 
place during the study period. It is therefore not possible to say what overall proportion of their consultations was for minor ailments. This fact limits, somewhat, the conclusions that can be drawn. In addition, GPs' views may have been subject to potential recall bias. GPs were asked to give their view in a single session on completion of data collection from their patients. This was to minimize the GP's time commitment and ensure that data were collected. Although it is likely that they would remember more clearly the consultations that had occurred most recently, neither GP expressed, nor displayed, difficulty in recalling their consultations during data collection.

The key strength of the methodology is that, for the first time, GP consultations have been placed explicitly and overtly in the minor ailment context. The meaning of the term 'minor ailment' was defined by the people who matter; patients experiencing illness and GPs being consulted. At no time during the study was the researchers' meaning imposed on the subjects, thereby adding to the validity of the results.

The findings from this study raise a number of potentially important issues both at individual GP level and for wider health policy. A mismatch between GPs' and patients' perceptions was identified in over $85 \%$ of cases. Given that previous research has identified, at individual consultation level, that GPs often perceived patients to be less ill than the patient did themselves (Martin et al., 1991), it is reasonable to expect that if the patient considered their ailment to be minor, their GP would concur. Furthermore, from a GP perspective 'minor ailment' and 'necessary consultation' would seem likely to be mutually exclusive terms. It would therefore appear reasonable to expect that the GPs would view the vast majority of all the patient-perceived minor ailment consultations to be unnecessary minor ailment consultations. However, in this study this was the case for only $30 \%$ of these consultations. The data identified that the availability of the appropriate management option and individual patient factors, including previous knowledge and experience of a condition and their need for reassurance may influence GPs' views. Indeed, this reinforces the view that GPs have a very valuable role to play in reassuring patients that they are, in fact, experiencing a minor ailment.

It was also surprising to find that over half of the patient perceived minor ailment consul- tations were considered by the GP not to be minor ailments. Although in terms of the key issue of appropriate management of illness, these patients had chosen an appropriate option they are an interesting and potentially important subgroup. These data support the supposition that some people clearly underestimate their illness and contradicts the view that patients with minor ailments consult unnecessarily.

This small study has opened up the underresearched area of both patients' and GPs' perceptions of minor ailments. Clearly from both the lay and professional perspective minor ailment consultations are more complex than just trivial, inappropriate or unnecessary consultations that waste the time of highly pressured GPs. From both perspectives this is far from the complete picture. Two distinct subgroups of minor ailments have been shown to exist; those that can and those that cannot be managed by the patient alone. Further work is undoubtedly required to unravel this complex area further. Furthermore, it raises the important question of whether the 'help' patients require or want, needs, in all cases, to be a GP consultation. Whether it could encompass seeking advice from other health professionals was not investigated. However, there is literature to support the view that nurses within GP practices (Horrocks et al., 2002) or community pharmacists (Hassell et al., 1997; Tully et al., 1997) potentially have a role to play as alternative sources of advice. As different patients experiencing the same minor ailment may prefer, or choose, to manage their condition in different ways, it is incumbent upon all health professionals to support patients in that decision-making process.

\section{References}

Bentzen, N., Christiansen, T. and Pedersen, K.M. 1989: Selfcare within a model for demand for medical care. Social Science Medicine 29, 185-93.

Cartwright, A. 1967: Patients and their doctors. A study of general practice. London: Routledge and Kegan Paul.

Cartwright, A. and Anderson, R. 1981: General practice revisited. A second study of patients and their doctors. Cambridge: Tavistock Publications.

Department of Health. 1999a: NHS healthcare advice direct to your living room. DoH press release, 1999/0738.

Department of Health. 1999b: NHS primary care walk-in centres. Health Services circular, 1999/116.

Freer, C.B. 1980: Self-care: A health diary study. Medical Care $18,853-61$. 
Hannay, D.R. 1979: The symptom iceberg: a study of community health. London: Routledge and Kegan Paul.

Hassell, K., Noyce, P.R., Rogers, A., Harris, J. and Wilkinson, J. 1997: A pathway to the GP: the pharmaceutical 'consultation' as a first port of call in primary health care. Family Practice 14, 498-502.

Horrocks, S., Anderson, A. and Salisbury, C. 2002: Systematic review of whether nurse practitioners working in primary care can provide equivalent care to doctors. British Medical Journal 324, 819-23.

Marsh, G.N. and Dawes, M.L. 1995: Establishing a minor illness nurse in a busy general practice. British Medical Journal 310, 778-80.

Martin, E., Russell, D., Goodwin, S., Chapman, R., North, M. and Sheridan, P. 1991: Why patients consult and what happens when they do. British Medical Journal 303, 289-92.

Morrell, D.C. 1976: Symptoms perceived and recorded by patients. Journal of the Royal College of General Practitioners 26, 398-403.

Scambler, A., Scambler, G. and Craig, D. 1981: Kinship and friendship networks and women's demand for primary care. Journal of the Royal College of General Practitioners 31, 746-50.

Shum, C., Humphreys, A., Wheeler, D., Cochrane, M., Skoda, S. and Clement, S. 2000: Nurse management of patients with minor illnesses in general practice: multicentre, randomized, controlled trial. British Medical Journal 320, 1038-43.

Tully, M.P., Hassell, K. and Noyce, P.R. 1997: Advice-giving in community pharmacies in the UK. Journal of Health Services Research and Policy 2, 38-50. 